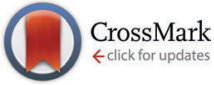

Cite this: Chem. Commun., 2016, 52,1586

Received 14th September 2015, Accepted 27th November 2015

DOI: $10.1039 / c 5 c c 07694 c$

www.rsc.org/chemcomm

\title{
Synthesis of glycosylphosphatidylinositol (GPI)-anchor glycolipids bearing unsaturated lipids $\dagger$
}

\author{
B.-Y. Lee, ${ }^{a}$ P. H. Seeberger ${ }^{\mathrm{ab}}$ and D. Varon Silva ${ }^{\mathrm{ab}}$
}

\begin{abstract}
2-Naphthyl-methyl ethers as permanent protecting groups are readily removed under acidic conditions and are key to the synthesis of complex glycosylphosphatidylinositol anchors containing unsaturated lipids. The total synthesis of the GPI pseudo-disaccharide core found on the surface of the Trypanosoma cruzi parasite serves to illustrate the power of the strategy.
\end{abstract}

GPIs are complex glycolipids that are ubiquitous in eukaryotic cells and have a common pseudo-pentasaccharide core structure 6-O-NEtP-Man $\alpha 1$-2-Man $\alpha 1-6-M a n \alpha 1-4-G l c N \alpha 1-6-I n o-1-P$ (Fig. 1). ${ }^{1}$ This conserved core is generally modified by additional phosphorylations, glycosylations, or acylations at the 2-O position of the myo-inositol in a cell-type dependent manner. ${ }^{2}$ GPIs are heterogeneous in the glycan as well as the lipid part. Inositol can bear $s n$-1-alkyl-2-acylglycerol, $s n$-1,2-diacylglycerol, or ceramide adorned with lipid chains of different lengths and degrees of saturation. ${ }^{3}$

Chagas disease, caused by the parasite Trypanosoma cruzi (T. cruzi), is a major public health problem in Latin America, infects around 7-8 million persons worldwide, and causes more than 10000 deaths each year. ${ }^{4}$ There is no vaccine for this disease and the two drugs available for treatment are used sparingly due to their cost, side effects, and low antiparasitic activity in patients with chronic infections. ${ }^{5}$

The cell surface of T. cruzi contains a high concentration of glycosylphosphatidylinositol (GPI) molecules, which exhibit proinflammatory activities comparable to bacterial lipopolysaccharides and are predominately attached to highly glycosylated mucins and phosphoglycans. ${ }^{6,7}$ Structural hallmarks of these GPIs are a glycan branch of galactoses, a $T$. cruzi specific 2-aminoethylphosphonate (2-AEP) unit at the 6-O position of the glucosamine residue and the presence of unsaturated fatty

\footnotetext{
${ }^{a}$ Max Planck Institute of Colloids and Interfaces, Biomolecular Systems, Am Mühlenberg 1, 14476 Potsdam, Germany.E-mail: daniel.varon@mpikg.mpg.de

${ }^{b}$ Department of Chemistry and Biochemistry, Freie Universität Berlin,

Arnimallee 22, 14195 Berlin, Germany

$\dagger$ Electronic supplementary information (ESI) available. See DOI: 10.1039/c5cc07694c
}

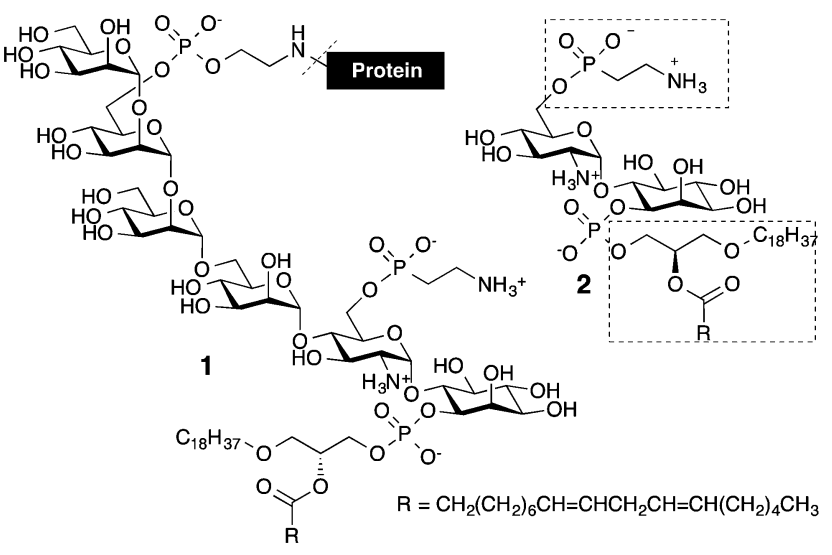

Fig. 1 Glycosylphosphatidylinositol anchor of the parasite Trypanosoma cruzi.

acids in the phospholipid, ${ }^{8,9}$ which have been associated with the biological activity of these molecules. ${ }^{6,10}$

Isolation of homogeneous GPIs is extremely difficult due to the heterogeneity and amphiphilic character of both the glycan and lipid. Biological evaluation of T. cruzi GPIs and potential applications for the diagnosis and prevention of Chagas disease require synthetic GPIs bearing unsaturated lipids. Most synthetic strategies for GPI glycolipids use benzyl ethers as permanent carbohydrate protecting groups. However, the reductive conditions required for benzyl ether removal are not compatible with the double bonds present in the lipid moiety. ${ }^{11}$ While this issue can be avoided through the use of benzoyl esters or PMB ethers, ${ }^{12,13}$ their use in this application has been limited due to the saponification of the fatty acid esters during base-mediated benzoyl ester removal and the low stability of PMB ethers under the mild acidic conditions commonly used for glycosylations. ${ }^{13-15}$

While the 2-naphthylmethyl ether (Nap) group has primarily been used as a temporary mask for hydroxyl groups in carbohydrate chemistry, ${ }^{16,17}$ its stability during glycosylation reactions and its orthogonality to silyl ethers, acyl esters, and even PMB ethers make it the ideal group for permanent protection during GPI synthesis. 
Herein, we report an efficient strategy for the synthesis of GPIs bearing unsaturated lipids. The key of our strategy is the use of stable 2-naphthylmethyl ethers for permanent hydroxyl group protection, which are readily removed using acidic conditions. The strategy is illustrated for the synthesis of a portion of the GPI anchor from T. cruzi.

We considered an assembly sequence based on our recently reported strategy for accessing GPIs with saturated lipids, in which the glycan is assembled first and the phosphorylations are installed at a late-stage of the synthesis. ${ }^{17,18}$ In this strategy, Nap ethers replaced the benzyl ethers as permanent protecting groups and the Allyl and PMB ethers were included as temporary protecting groups for positions requiring phosphorylation, while an azide served as a masked amine.

To obtain the bis-phosphorylated GPI pseudo-disaccharide 2, a number of building blocks were envisioned branching from the core glycan fragment 3 containing Nap ethers as permanent protecting groups. For the subsequent phosphorylations, building blocks 4 and 5 were necessitated (Fig. 2). To assemble the glycan moiety, building blocks 6 and 7 were designed to generate the desired $\alpha$-glucosylated myo-inositol. Through a series of protecting group manipulations, the pseudo-disaccharide glycan GlcN-Ino 3 was obtained with both a PMB and an allyl-protecting group. Selective step-wise removal of the PMB and allyl ethers, followed by phosphorylations and global deprotection, will complete the synthesis.

Synthesis of the protected optically pure D-myo-inositol building block 7 started from methyl-glucoside. ${ }^{19,20}$ The primary alcohol was protected with a trityl group, followed by the overnight per-naphthyl-methylation using 2-(naphthyl)-methyl bromide and $\mathrm{NaH}$ (Scheme 1). After removal of the trityl ether, alcohol oxidation with $\mathrm{SO}_{3}$-pyridine complex to the aldehyde, and subsequent acetylation produced enol-ester 10. A mercury II-mediated Ferrier type-2 rearrangement afforded intermediate $\mathbf{1 1}$ in good yield $(68 \%)$. Stereoselective reduction of ketone 11 with $\mathrm{NaBH}(\mathrm{OAc})_{3}$ followed by deacetylation under Zemplen conditions delivered triol 12. Regioselective allylation of $\mathbf{1 2}$ using bis(tributyltin) oxide and the selective protection at $\mathrm{O}-2$ as a napthyl-methyl ether gave building block 7 in 19\% overall yield. The Nap group did not affect the process when compared to benzyl ethers and delivered the protected myo-inositol with improved yield. ${ }^{20}$

For the synthesis of GlcN-Ino pseudo-disaccharide 3 , the required glucosamine donor 6 was obtained from glucosamine azide 13 by transformation of the anomeric acetyl group into a

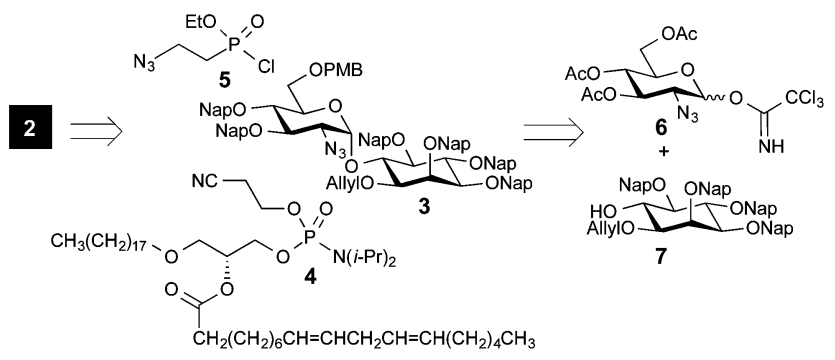

Fig. 2 Retrosynthetic analysis of the T. cruzi GPI pseudo-disaccharide containing an unsaturated lipid chain.

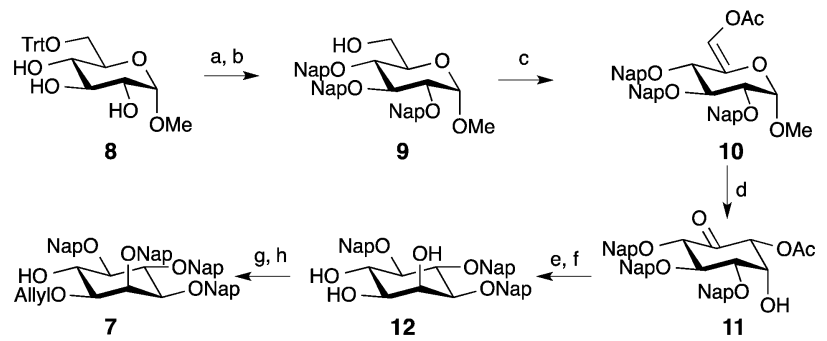

Scheme 1 Synthesis of protected myo-inositol 7. Reaction conditions: (a) $\mathrm{NapBr}$, NaH, DMF, rt, overnight, 85\%; (b) p-TsOH, MeOH, rt, 16 h, 90\%; (c) i. $\mathrm{SO}_{3}-\mathrm{Py}, \mathrm{DIPEA}, \mathrm{DMSO}, \mathrm{CH}_{2} \mathrm{Cl}_{2}, \mathrm{O}^{\circ} \mathrm{C}, 1 \mathrm{~h}$, ii. $\mathrm{Ac}_{2} \mathrm{O}, \mathrm{K}_{2} \mathrm{CO}_{3}, \mathrm{MeCN}$, reflux, 4 h, 80\% (2 steps); (d) i. $\mathrm{Hg}(\mathrm{OTf})_{2}$, acetone $/ \mathrm{H}_{2} \mathrm{O}, \mathrm{rt}, 1 \mathrm{~h}$, ii. $\mathrm{NaOAc}, \mathrm{NaCl}$, $0{ }^{\circ} \mathrm{C}$ to rt, $12 \mathrm{~h}$; (e) $\mathrm{NaBH}(\mathrm{OAc})_{3}, \mathrm{MeCN}, \mathrm{AcOH}, \mathrm{rt}, 12 \mathrm{~h}, 68 \%$ (2 steps); (f) $\mathrm{NaOMe}, \mathrm{MeOH}, \mathrm{rt}, 30 \mathrm{~min}$; (g) i. $\left(\mathrm{Bu}_{3} \mathrm{Sn}\right)_{2} \mathrm{O}$, toluene, reflux, $5 \mathrm{~h}$, ii. AllBr, TBAl, toluene $65^{\circ} \mathrm{C}, 17 \mathrm{~h}, 68 \%$ (20\% recovered starting material); (h) NapBr, $\mathrm{NaH}, \mathrm{DMF},-20{ }^{\circ} \mathrm{C}$ to $0{ }^{\circ} \mathrm{C}, 2 \mathrm{~h}, 67 \%$.

trichloroacetimidate using a two-step protocol. Three different conditions were tested to obtain the desired $\alpha$-glycosidic linkage. ${ }^{21}$ Product 14 was obtained in the best $\alpha / \beta$ ratio $(11: 1)$ at room temperature, using $\mathrm{Et}_{2} \mathrm{O} / \mathrm{CH}_{2} \mathrm{Cl}_{2}$ as solvent and TMSOTf as an activator (Scheme 2).

After separation of the $\alpha / \beta$ isomers of $\mathbf{1 4}$ by silica gel chromatography, the acetyl groups were removed using Zemplen conditions and the 4-O and 6-O hydroxyls were protected as a $p$-methoxybenzylidene acetal by reacting the generated triol with anisaldehyde dimethylacetal under acidic conditions. Nap protection of the free 3-O position of disaccharide $\mathbf{1 5}$, followed by selective opening of the 4,6-acetal group using $\mathrm{NaCNBH}_{3}$ under acidic conditions, and protection of the 4-O position delivered the fully protected 3 in good yield (Scheme 2).

With the glycan part in hand, the synthesis of the phosphorylated building blocks 4 and $\mathbf{5}$ was advanced. Etherification of alcohol 17 using 1-bromooctadecane and $\mathrm{NaH}$, followed by the hydrolysis of the isopropylidene acetal and subsequent protection of the primary alcohol using a TBS group furnished 19 (Scheme 3a).

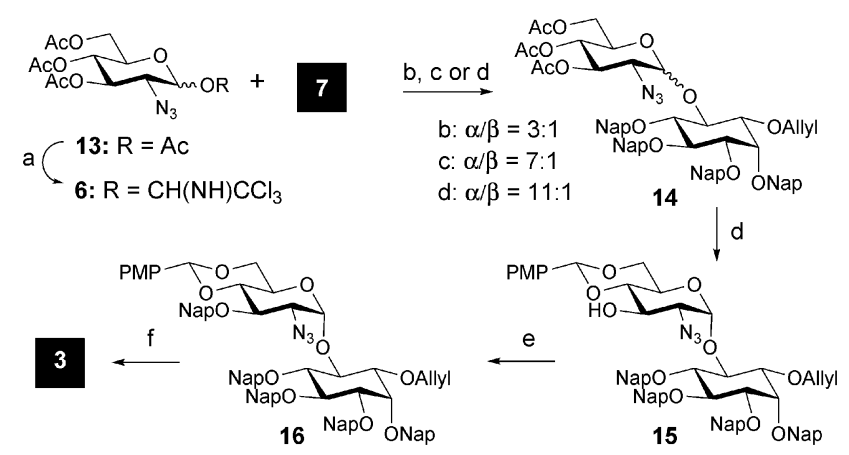

Scheme 2 Synthesis of Glc-Ino pseudo-disaccharide 3. Reaction conditions: (a) i. $\mathrm{NH}_{3}(\mathrm{~g}), \mathrm{CH}_{3} \mathrm{CN}, 0{ }^{\circ} \mathrm{C}$ to rt, $1 \mathrm{~h}$, ii. $\mathrm{CCl}_{3} \mathrm{CN}, \mathrm{DBU}, \mathrm{CH}_{2} \mathrm{Cl}_{2}, 0{ }^{\circ} \mathrm{C}, 15 \mathrm{~min}(\alpha / \beta$ mixture donor, for $\mathrm{b}$ and $\mathrm{c}$ ) or $\mathrm{CCl}_{3} \mathrm{CN}, \mathrm{K}_{2} \mathrm{CO}_{3}, \mathrm{CH}_{2} \mathrm{Cl}_{2}$, rt, $5 \mathrm{~h}$ ( $\beta$ donor, for d); (b)TMSOTf, $\mathrm{Et}_{2} \mathrm{O} / \mathrm{CH}_{2} \mathrm{Cl}_{2}(6: 1), 0{ }^{\circ} \mathrm{C}, 1 \mathrm{~h}, 87 \%$; (c) TMSOTf, $\mathrm{Et}_{2} \mathrm{O} / \mathrm{CH}_{2} \mathrm{Cl}_{2}(6: 1), \mathrm{rt}$, $1 \mathrm{~h}, 85 \%$; (d) TMSOTf, $\mathrm{CH}_{2} \mathrm{Cl}_{2}$, rt, $30 \mathrm{~min}, 91 \%$; (e) i. $\mathrm{NaOMe}, \mathrm{MeOH}$, rt, 1 h, ii. Anisaldehyde dimethylacetal, CSA, DMF, rt, overnight, 86\% (2 steps); (f) $\mathrm{NapBr}$, $\mathrm{NaH}$, TBAl, DMF, rt, overnight, $88 \%$; (g) $\mathrm{NaCNBH}_{3}$, TFA, THF/CH $\mathrm{CH}_{2}$, rt, 9 h, $80 \%$; (h) $\mathrm{NapBr}, \mathrm{NaH}, \mathrm{DMF}$, rt, overnight, 82\%. PMP: $p$-methoxyphenyl. 


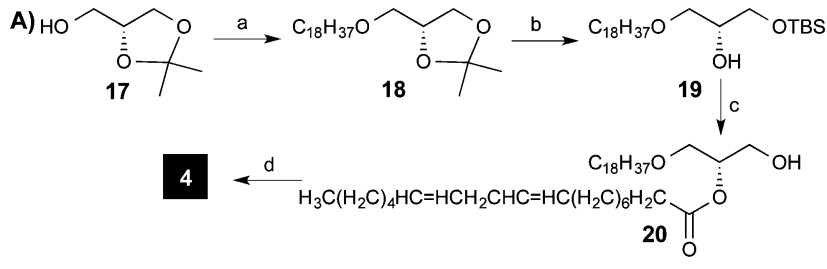

B)

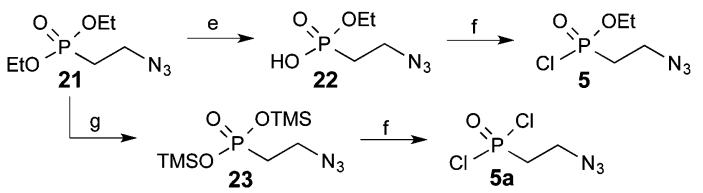

Scheme 3 Synthesis of phosphorylation synthons. Reagents and conditions: (a) 1-Bromooctadecane, $\mathrm{NaH}$, Toluene, $97 \%$; (b) i. $\mathrm{HCl}, \mathrm{MeOH}, 15 \%$, ii. $\mathrm{TBSCl}$, Imidazole, DMF, 75\%; (c) i. linoleic acid, DCC, DMAP, $\mathrm{CH}_{2} \mathrm{Cl}_{2}$, r.t., ii. $\mathrm{BF}_{3}-$ $\mathrm{Et}_{2} \mathrm{O}, \mathrm{CH}_{2} \mathrm{Cl}_{2}, 0{ }^{\circ} \mathrm{C}, 60 \%$ (over two steps); (d) bis(diisopropylamino)(2cyanoethoxy)phosphine, $1 \mathrm{H}$-tetrazole, $\mathrm{CH}_{2} \mathrm{Cl}_{2}, 90 \%$; (e) $\mathrm{LiBr}$, 2-pentanone, $110{ }^{\circ} \mathrm{C}, 2$ h, quant.; (f) Oxalylchloride, DMF cat., $\mathrm{CH}_{2} \mathrm{Cl}_{2}, \mathrm{rt}, 1 \mathrm{~h}$; (g) $\mathrm{TMSBr}$, $\mathrm{CH}_{2} \mathrm{Cl}_{2}, \mathrm{O}^{\circ} \mathrm{C}$ to rt, overnight.

Acylation of alcohol 19 with linoleic acid using DCC/DMAP and deprotection of the silyl group provided the desired alkylacylglycerol 20 (Scheme 3). To obtain the required phosphoamidite building block, glycerol derivative 20 was transformed into 4 using the commercially available bis(diisopropylamino)(2-cyanoethoxy)phosphine and $1 H$-tetrazole. ${ }^{22}$

Ethyl-(2-azidoethyl)phosphonochloridate (5) and bis(chloro)(2-azidoethyl)phosphonate (5a) were obtained from commercially available diethyl (2-bromoethyl)phosphonate. Starting with the conversion of bromide into the corresponding azide $\mathbf{2 1},{ }^{12}$ the obtained ethyl phosphonate 21 was converted into chlorophosphonate $\mathbf{5}$ using a two-step protocol. First, ethylphosphonate 21 was hydrolyzed with $\mathrm{LiBr}$ to provide phosphonic acid mono ethyl ester $22,{ }^{23}$ which was converted into 5 by treatment with oxalylchloride. $^{24}$ Phosphonodichloridate 5 a was synthesized from ethyl phosphonate $\mathbf{2 1}$ via silylated intermediate 23, which underwent reaction with oxalyl chloride. ${ }^{25}$

Two phosphorylation sequences of 3 were evaluated. In the first case, the PMB group of pseudo-disaccharide 3 was selectively removed under acidic conditions and without affecting the Nap groups to obtain alcohol $24 .{ }^{16}$ This compound was further phosphitylated with 5 in the presence of $1 H$-tetrazole and DIPEA. Following reduction of the azides using DTT/DIPEA and amine protection with Boc anhydride the phosphonate compound 26 was delivered, which was deallylated using $\mathrm{PdCl}_{2}$ and NaOAc in AcOH. To complete the synthesis of the fully modified pseudodisaccharide, the free hydroxyl group of $\mathbf{2 7}$ was phosphitylated with $\mathbf{4}$ and oxidized to the corresponding phosphorylated product 28 by using tert-butylhydroperoxide (Scheme 4).

Removal of the protecting groups from 2-aminoethylphosphonate proved to be more challenging than expected. High stability of the phosphorous ethyl ester in $\mathbf{2 8}$ was observed after reduction of the azide and Boc protection of the formed amines. A variety of conditions were evaluated for the removal of the ester (see ESI $\dagger$ ); however they resulted in non-reaction or in the decomposition of the starting material when long reaction times and strong reaction conditions were used. As such, an

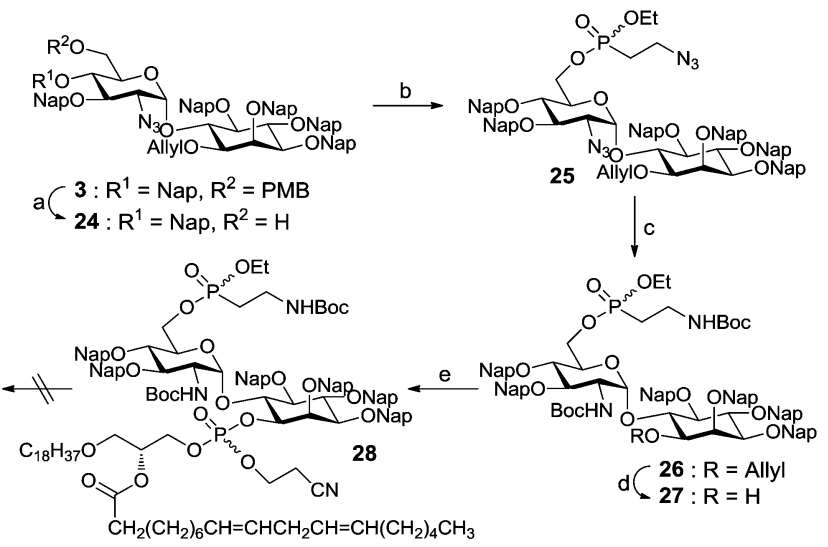

Scheme 4 Reagents and conditions: (a) $15 \% \mathrm{TFA}, \mathrm{CH}_{2} \mathrm{Cl}_{2}, 0{ }^{\circ} \mathrm{C}, 15 \mathrm{~min}$, 91\%; (b) 5, DIPEA, $1 \mathrm{H}$-tetrazole, toluene, $0{ }^{\circ} \mathrm{C}$ to $\mathrm{rt}$, overnight, $72 \%$; (c) i. dithiothreitol, DIPEA, MeCN/ $\mathrm{H}_{2} \mathrm{O}, \mathrm{rt}, 2 \mathrm{~h}$, ii. $\mathrm{Boc}_{2} \mathrm{O}, \mathrm{DIPEA}, \mathrm{MeOH}, \mathrm{rt}, 2 \mathrm{~h}$, 86\% (2 steps); (d) $\mathrm{PdCl}_{2}, \mathrm{NaOAc}, \mathrm{AcOH}, \mathrm{rt}, 6$ h, 71\%; (e) i. 4, $1 \mathrm{H}$-tetrazole, $\mathrm{CH}_{2} \mathrm{Cl}_{2} / \mathrm{MeCN}, \mathrm{rt}, 5 \mathrm{~h}$, ii. $t-\mathrm{BuOOH}, \mathrm{Me}_{2} \mathrm{~S},-40{ }^{\circ} \mathrm{C}, 2 \mathrm{~h}, 64 \%$ (2 steps).

alternative assembly/deprotection sequence was needed. First, the allyl group of pseudo-disaccharide 3 was removed in a two-step protocol involving an iridium catalyzed isomerization and hydrolysis with mercury chloride. The resulting free hydroxyl group was phosphitylated with phosphoamidite 4 and oxidized with $t$ - $\mathrm{BuOOH}$ to deliver phospholipidated pseudo-dissacharide $\mathbf{3 0}$.

To install the 2-azidoethylphosphonate unit, the PMB on the glucosamine of $\mathbf{3 0}$ was selectively removed under acidic conditions using 15\% TFA in DCM (Scheme 5). Then the hydroxyl group was reacted with 5a using $1 H$-tetrazole and DIPEA activation at $0{ }^{\circ} \mathrm{C}$, followed by the hydrolysis of the remaining chloride by addition of water. The remaining protecting groups on the fully decorated pseudo-disaccharide were removed via a three-step protocol. Efficient removal of the cyanoethoxy group of the phospholipid with DBU was followed by azide reduction with zinc in AcOH. Different conditions were evaluated for the final removal of the Nap groups. Methods involving oxidative conditions (DDQ) delivered a mixture of compounds due to incomplete deprotection. However, by using TFA/toluene $8: 1$ at $0{ }^{\circ} \mathrm{C}$, target 2 was obtained in high yield ( $72 \%$ for the three deprotection steps) after purification using size exclusion chromatography. ${ }^{26}$

In summary, a new strategy to obtain glycosylphosphatidylinositol anchors containing unsaturated lipid chains has been disclosed. The strategy is based on the use of 2-napthyl-methyl ethers for permanent protection and allyl and PMB ethers as orthogonal groups for masking positions requiring late stage modifications. The synthesis of the required building blocks demonstrated that the size of the Nap group did not affect the reactivity of donors and acceptors and can easily replace benzyl ether in established protocols. We described the synthesis of the complex Glc-Ino pseudo-disaccharide core of the T. cruzi parasite GPI, which bears a lipid moiety, and is modified by a linoleic acid ester and a 2-aminophosphoethylamine. We demonstrated the high stability of the Nap group in glycosylation and phosphorylation reactions and its removal under acidic conditions without affecting the phosphorylations, the alkyl ether on alkylacylglycerol, or the fatty 


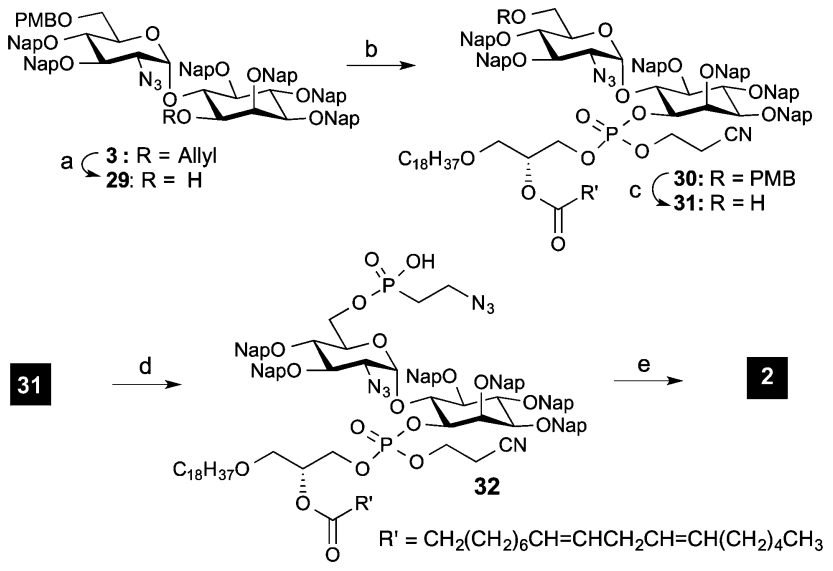

Scheme 5 Reagents and conditions: (a) i. $\operatorname{Ir}(\mathrm{COD})_{2} \mathrm{Cl}_{2}, \mathrm{THF}, \mathrm{rt}, 5 \mathrm{~h}, \mathrm{ii}$. $\mathrm{HgCl}_{2}$ $\mathrm{HgO}$, acetone, rt, $1 \mathrm{~h}, 91 \%$; (b) i. $4,1 \mathrm{H}$-tetrazole, $\mathrm{CH}_{2} \mathrm{Cl}_{2}$, rt, ii. $t-\mathrm{BuOOH}, \mathrm{Me}_{2} \mathrm{~S}$, $-40{ }^{\circ} \mathrm{C}, 2 \mathrm{~h}, 87 \%$ (2 steps); (c) TFA, $\mathrm{CH}_{2} \mathrm{Cl}_{2}, 0{ }^{\circ} \mathrm{C}, 80 \%$; (d) i. 5a, $1 \mathrm{H}$-tetrazole, DIPEA, toluene, $0{ }^{\circ} \mathrm{C}, 2 \mathrm{~h}$, ii. $\mathrm{H}_{2} \mathrm{O}, \mathrm{rt}, 1 \mathrm{~h}, 87 \%$; (e) i. $\mathrm{DBU}, \mathrm{CH}_{2} \mathrm{Cl}_{2}$, rt, ii. $\mathrm{Zn}, \mathrm{AcOH}$, $\mathrm{CH}_{2} \mathrm{Cl}_{2}$, rt, iii. TFA, toluene, $0{ }^{\circ} \mathrm{C}, 72 \%$ (calculated from 32 ).

acid containing the double bond. This strategy will enable the synthesis of diverse glycosylphosphatidylinositols and other glycolipids requiring unsaturated lipids and give access to pure GPIs for biological investigation.

We thank the Max Planck Society and the RIKEN-Max Planck Joint Center for Systems Chemical Biology for financial support. We thank Ankita Malik for her support in the deprotection and characterization of the final compound.

\section{Notes and references}

1 M. G. Paulick and C. R. Bertozzi, Biochemistry, 2008, 47, 6991-7000. 2 M. A. J. Ferguson and A. F. Williams, Annu. Rev. Biochem., 1988, 57, 285-320.

3 M. J. McConville and M. A. Ferguson, Biochem. J., 1993, 294, 305-324. 4 A. Rassi Jr, A. Rassi and J. A. Marin-Neto, Lancet, 2010, 375, 1388-1402. 5 E. Chatelain, J. Biomol. Screening, 2015, 20, 22-35.
6 I. C. Almeida, M. M. Camargo, D. O. Procopio, L. S. Silva, A. Mehlert, L. R. Travassos, R. T. Gazzinelli and M. A. J. Ferguson, EMBO J., 2000, 19, 1476-1485.

7 R. M. de Lederkremer, C. Lima, M. I. Ramirez, M. A. Ferguson, S. W. Homans and J. Thomas-Oates, J. Biol. Chem., 1991, 266, $23670-23675$.

8 A. A. Serrano, S. Schenkman, N. Yoshida, A. Mehlert, J. M. Richardson and M. A. Ferguson, J. Biol. Chem., 1995, 270, 27244-27253.

9 J. O. Previato, C. Jones, M. T. Xavier, R. Wait, L. R. Travassos, A. J. Parodi and L. Mendonca-Previato, J. Biol. Chem., 1995, 270, $7241-7250$

10 M. M. Camargo, I. C. Almeida, M. E. Pereira, M. A. Ferguson, L. R. Travassos and R. T. Gazzinelli, J. Immunol., 1997, 158, 5890-5901.

11 Y. H. Tsai, X. Y. Liu and P. H. Seeberger, Angew. Chem., Int. Ed., 2012, 51, 11438-11456.

12 D. V. Yashunsky, V. S. Borodkin, M. A. J. Ferguson and A. V. Nikolaev, Angew. Chem., Int. Ed., 2006, 45, 468-474.

13 B. M. Swarts and Z. W. Guo, J. Am. Chem. Soc., 2010, 132, 6648-6650.

14 B. M. Swarts and Z. W. Guo, Chem. Sci., 2011, 2, 2342-2352.

15 L. Yan and D. Kahne, Synlett, 1995, 523-524.

16 J. Xia, S. A. Abbas, R. D. Locke, C. F. Piskorz, J. L. Alderfer and K. L. Matta, Tetrahedron Lett., 2000, 41, 169-173.

17 M. J. Gaunt, J. Yu and J. B. Spencer, J. Org. Chem., 1998, 63, 4172-4173.

18 A. Lipták, A. Borbás, L. Jánossy and L. Szilágyi, Tetrahedron Lett., 2000, 41, 4949-4953.

19 Z. J. Jia, L. Olsson and B. Fraser-Reid, J. Chem. Soc., Perkin Trans. 1, 1998, 631-632.

20 X. Y. Liu, B. L. Stocker and P. H. Seeberger, J. Am. Chem. Soc., 2006, 128, 3638-3648.

21 Y.-H. Tsai, S. Gotze, I. Vilotijevic, M. Grube, D. Varon Silva and P. H. Seeberger, Chem. Sci., 2013, 4, 468-481.

22 H. Q. Nguyen, R. A. Davis and J. Gervay-Hague, Angew. Chem., Int. Ed., 2014, 53, 13400-13403.

23 D. Sikora, T. Nonas and T. Gajda, Tetrahedron, 2001, 57, 1619-1625.

24 H. P. Dijkstra, H. Sprong, B. N. H. Aerts, C. A. Kruithof, M. R. Egmond and R. J. M. K. Gebbink, Org. Biomol. Chem., 2008, 6, 523-531.

25 L. Rigger, R. L. Schmidt, K. M. Holman, M. Simonovic and R. Micura, Chem. - Eur. J., 2013, 19, 15872-15878.

26 During the preparation of this report a new method for the acidic removal of the Nap group was published. Although we did not try this method in our compounds, it is an additional method for global deprotection. see: A. G. Volbeda, H. A. V. Kistemaker, H. S. Overkleeft, G. A. van der Marel, D. V. Filippov and J. D. C. Codée, J. Org. Chem., 2015, 80, 8796-8806. 